\title{
Stochastic Aspects of the Force Network in a Regular Granular Piling
}

\author{
Christophe Eloy $\left({ }^{*}\right)$ and Éric Clément $\left({ }^{* *}\right)$ \\ Laboratoire des Milieux Désordonnés et Hétérogènes $\left({ }^{* *}\right)$, B. 86, \\ Université Pierre et Marie Curie, 4 Place Jussieu, 75252 Paris Cedex 05, France
}

(Received 14 May 1997, received in final form 25 July 1997, accepted 5 August 1997)

PACS. $46.10+z$ - Mechanics of discrete systems

PACS.05.40. $+\mathrm{j}$ - Fluctuation phenomena, random processes, and Brownian motion

PACS.83.70.Fn - Granular solids

\begin{abstract}
We study the influence of solid friction on the structure of the force network sustaining a regular bidimensional granular piling. We show how the mechanical equations describing local equilibrium may couple naturally with a stochastic variable. A Monte Carlo method is designed to extract the force networks satisfying static equilibrium conditions. A statistical ensemble is constructed and we study some of its structural and stochastic properties. We address, in particular, the question of the vertical load transfer in two different cases $\imath . e$. the response to a localized force excess at the top of an horizontal piling and the distribution of forces under a sand pile. Using this very simple mechanical model, we test the validity of the passage to various stochastic descriptions as well as the existence of macroscopic constitutive relations. We also address the question of the influence of a local friction bias on the macroscopic static equilibrium conditions.
\end{abstract}

\section{Introduction}

The static mechanical properties of an assembly of non cohesive grains is a long standing difficult problem. Since the pioneering experimental work of Dantu [1] (see also [2]), who visualized large fluctuations and long range disordered structures of the force network, it has been recognized that the passage to a continuous medium description would be an arduous task. More recently, numerical simulations based on various algorithmic principles [3-7] have evidenced the highly disordered character of the contact force distribution. Recent theoretical works have proposed, on the base of phenomenology and symmetry properties, new sets of continuous media equations describing transport of contact forces between the grains. These approaches introduce, in a simple way, relevant granular features such as the directional propagation of forces and also the existence of arches whenever a boundary is considered [8-10]; they come as an alternative viewpoint to the standard way to close the mechanical equation in the static

( $\left.{ }^{*}\right)$ Now at IRPHE, 12 avenue du Général Leclerc, 13003 Marseille, France

$\left({ }^{* *}\right)$ Author for correspondence (e-mail erc@ccr.jussieu.fr)

$\left({ }^{* * *}\right)$ URA 800 CNRS

(C) Les Éditions de Physique 1997 
or quasi static situations, usually based on the assumption of local incipient failure [11]. But so far, no explicit connection to fluctuating properties has been made. On the other hand, an original point of view is proposed by Liu et al. [12] (see also Coppersmith et al. [13]) assuming a simple but suggestive stochastic representation of the force redistribution from one layer of grains to the other. This model was designed to explain experimental measurements on the vertical force distribution at the bottom of a three-dimensional container filled with grains. Following this work, numerous stochastic models have been proposed in order to render the disordered character of the force propagation problem in a granular assembly $[14,15]$. The problem is still the validation of these models on the basic point of view of mechanical equations. Here, we present some results obtained on the force distribution of regular, bidimensional, arrays of grains. A fundamental aspect is the presence of static friction between the grains which renders the problem multi-valued in the sense of the Coulomb representation of solid contacts. This is a direct consequence of the Coulomb inequalities describing static equilibrium. We purposely do not enter in a more refined description of the contact status that could be based, for example, on a microscopic modelization of the real surfaces in contact which is a difficult issue indeed (see for example $[16,17]$ ). This is the reason why we stay in the frame work of the macroscopic Coulomb modelization. We use a Monte Carlo method to remove the solution degeneracies and we extract sets of exact solutions for the forces distribution. Therefore, a statistical study of the force networks is possible and a bridge towards a stochastic approach is discussed on rational mechanical grounds [18]. In this context, we investigate the statistical properties of the force network, the response to a local force excess and the problem of the stress distribution below a sand pile. We also address the question of constitutive relations relating the stress tensor components.

\section{Description of the Model}

The piling we consider here is made of a mono-disperse and bidimensional array of hard cylinders with a size and weight unity, piled in a triangular compact fashion (bidimensional cannon ball piling). This system would be close to the experimental set-up investigated by Travers et al. [2] for regular cylinders. The contact is assumed to be at a constant angle $\theta=60^{\circ}$ (see Fig. 1a) thus, each bead receives two forces of contact from the upper layer and distributes two contact forces to the layer downwards (See Fig. 1b). In this model, we explicitly neglect the presence of active contacts between beads at the same depth, this situation would be consistent with an angle of contact $\theta$ slightly below $60^{\circ}$. Each locus of arriving and departing forces is called a vertex. In this case, the structure of each vertex is quite simple but it is easy to imagine that this notion might be generalized according to general disordered geometrical properties of a granular contact network [19]. The contact forces are constrained by the solid friction properties of the material captured in a static coefficient $\mu$ defined in the Coulomb sense.

The force system acting on one bead is represented in Figures $1 b, c$. The upper cases letters stand for the forces upwards and the lower case letters stand for the forces downwards. The system of equations describing static equilibrium of the central bead is:

$$
\begin{gathered}
\left(-N_{1}-N_{2}+n_{1}+n_{2}\right) \sin \theta+\left(T_{1}-T_{2}-t_{1}+t_{2}\right) \cos \theta=1 \\
\left(-N_{1}+N_{2}+n_{1}-n_{2}\right) \cos \theta+\left(-T_{1}-T_{2}+t_{1}+t_{2}\right) \sin \theta=0 \\
T_{1}+T_{2}+t_{1}+t_{2}=0 .
\end{gathered}
$$

Note that we consider here not only equilibrium for the two degrees of translation but also explicatly the local rotational degree of freedom. This is usually expressed in the standard 


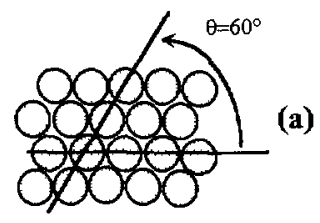

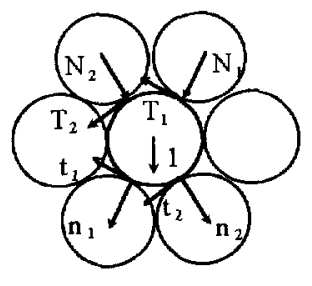

(b)

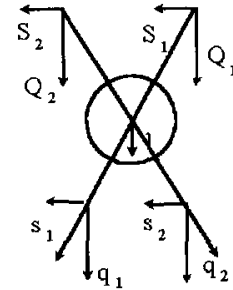

(c)

Fig. 1. - Description of the force model. a) Sketch of the piling structure. b) Contact forces, the arrows indicates the local positive axes. c) Horizontal and vertical charges.

mechanical equations of continuum media, at a coarsed grained level, via the symmetry of the stress tensor $\left(\left|\sigma_{x z}\right|=\left|\sigma_{z x}\right|\right)$. This condition, though necessary, might not be sufficient to provide a relevant description of static equilibrium for a granular assembly which is notoriously discontinuous at the granular level.

Now, we express the vertical and horizontal charges as a function of normal and tangential contact forces. We have:

$$
\begin{array}{ll}
q_{1}=n_{1} \sin \theta-t_{1} \cos \theta: & \text { vertical force transmitted on bead } 1 \text { down, } \\
q_{2}=n_{2} \sin \theta+t_{2} \cos \theta: & \text { vertical force transmitted on bead } 2 \text { down, } \\
s_{1}=n_{1} \cos \theta+t_{1} \sin \theta: & \text { horizontal force transmitted on bead } 1 \text { down, } \\
s_{2}=-n_{2} \cos \theta+t_{2} \sin \theta: & \text { horizontal force transmitted on bead } 2 \text { down, }
\end{array}
$$

for the beads upwards, identical relations hold with upper case letters.

The core of the statistical model of Liu et al. [12] is that each grain redistributes between the two neighbors downwards, the total amount of charge it has received from the layer just above. We show to which extend this redistribution can be described in a simple stochastic fashion. A fundamental quantity to monitor is the total vertical charge $C$ received by the central bead (see Fig. 1c). The charges received from the top beads 1 and 2 are respectively $Q_{1}$ and $Q_{2}$ and the charges transmitted on the beads 1 and 2 downwards are $q_{1}$ and $q_{2}$ respectively. We have:

$$
C=Q_{1}+Q_{2}+1=q_{1}+q_{2}
$$

Here, we assume the presence of gravity with a value of single bead weight taken as unity. Another quantity is the horizontal charge $\Sigma$, z.e. the projection of the contact forces received from the top beads on the horizontal direction, respectively $S_{1}$ and $S_{2}$. These forces are transmitted on the beads downwards, respectively $s_{1}$ and $s_{2}$ and we have:

$$
\Sigma=S_{1}+S_{2}=s_{1}+s_{2} \text {. }
$$


Therefore, in the horizontal and vertical charges representation, the equation system (1) yields the following set of three equations and four unknowns $\left(q_{1}, q_{2}, s_{1}, s_{2}\right)$ :

$$
\begin{aligned}
s_{1}+s_{2} & =S_{1}+S_{2} \\
q_{1}+q_{2} & =Q_{1}+Q_{2}+1 \\
q_{1}-q_{2}-\left(s_{1}+s_{2}\right) \tan \theta & =Q_{2}-Q_{1}+\left(S_{1}+S_{2}\right) \tan \theta .
\end{aligned}
$$

From this system of equations, it appears that a natural choice for a free parameter would be $p=s_{1}-s_{2}$, and provided this parameterization, $q_{1}, q_{2}, s_{1}$ and $s_{2}$ are perfectly determined by the equation set:

$$
\begin{aligned}
q_{1} & =Q_{2}+\frac{1}{2}+\tan \theta\left(S_{1}+S_{2}\right) \\
q_{2} & =Q_{1}+\frac{1}{2}-\tan \theta\left(S_{1}+S_{2}\right) \\
s_{1} & =\frac{1}{2}\left(S_{1}+S_{2}+p\right) \\
s_{2} & =\frac{1}{2}\left(S_{1}+S_{2}-p\right) .
\end{aligned}
$$

From the two first equations, it appears that the values of $q_{1}$ and $q_{2}$ depend explicitly on the known values of $Q_{1}, Q_{2}, S_{1}, S_{2}$ (and not on the arbitrary choice of $p$ ). Moreover, the choice of the parameter $p$ for each contact vertex cannot be made at random. It is limited by two physical constraints. The first is due to the unilaterality of contact force, i.e. $n_{1} \geq 0$ and $n_{2} \geq 0$. Second is the constraint for the contact forces to be contained within the Coulomb angle in order to define a static mechanical problem, z.e. $\left|t_{1}\right|<\mu n_{1}$ and $\left|t_{2}\right|<\mu n_{2}$.

The unilaterality conditions yield:

$$
\begin{gathered}
n_{1} \geq 0 \Rightarrow p \geq-2 \tan \theta Q_{2}-\tan \theta-\left(1+2 \tan ^{2} \theta\right)\left(S_{1}+S_{2}\right) \\
n_{2} \geq 0 \Rightarrow p \geq-2 \tan \theta Q_{1}-\tan \theta+\left(1+2 \tan ^{2} \theta\right)\left(S_{1}+S_{2}\right) .
\end{gathered}
$$

In the case where the friction coefficient $\mu<\tan \theta$, the statisticity conditions are for the contact 1:

$$
t_{1}<\mu n_{1} \Rightarrow p<\frac{\left(\left(1+2 \tan ^{2} \theta\right) \mu+\tan \theta\right)\left(S_{1}+S_{2}\right)+2(\mu \tan \theta+1) Q_{2}+\mu \tan \theta+1}{\tan \theta-\mu}
$$

$$
t_{1}>-\mu n_{1} \Rightarrow p>\frac{\left(-\left(1+2 \tan ^{2} \theta\right) \mu+\tan \theta\right)\left(S_{1}+S_{2}\right)+2(-\mu \tan \theta+1) Q_{2}-\mu \tan \theta+1}{\tan \theta+\mu}
$$

and for the contact 2 :

$$
\begin{gathered}
t_{2}>-\mu n_{2} \Rightarrow p<\frac{-\left(\left(1+2 \tan ^{2} \theta\right) \mu+\tan \theta\right)\left(S_{1}+S_{2}\right)+2(\mu \tan \theta+1) Q_{1}+\mu \tan \theta+1}{\tan \theta-\mu} \\
t_{2}<\mu n_{2} \Rightarrow p>\frac{\left(\left(1+2 \tan ^{2} \theta\right) \mu-\tan \theta\right)\left(S_{1}+S_{2}\right)+2(-\mu \tan \theta+1) Q_{1}-\mu \tan \theta+1}{\tan \theta+\mu} .
\end{gathered}
$$

Any set of contact forces which satisfies conditions of equation (5) provided the inequalities of equations $(6,7,8)$, defines a set of mechanically acceptable solutions for a vertex parametrized by $p$. 

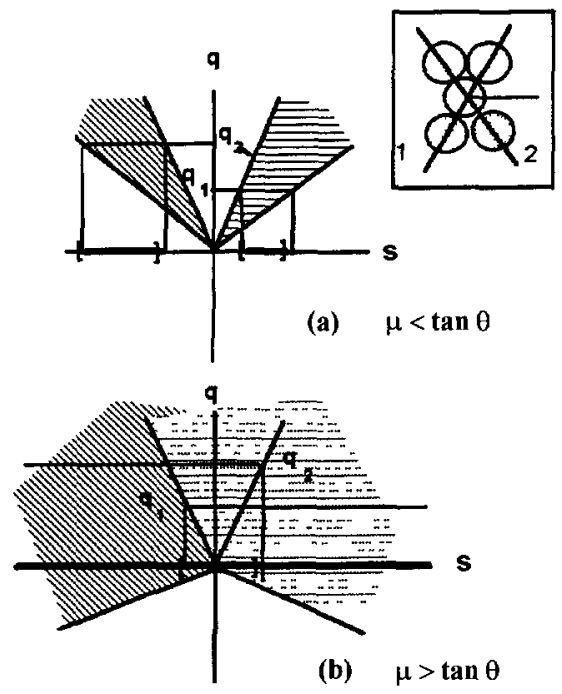

Fig. 2. - Representation of the horizontal and vertical charge transmission in the $q-s$ space. The inset displays the geometrical configuration. a) sketches a low friction situation and b) sketches a high friction configuration.

Note that this set may be empty for some values of $Q_{1}, Q_{2}, S_{1}, S_{2}$, meaning that inequations $(6,7,8)$ cannot be satisfied simultaneously. A mechanically acceptable solution has a simple geometrical representation captured in Figure 2. In this picture, we display the contact forces for beads 1 and 2 below (see inset) in the $(q, s)$ space. The choice of $s_{1}$ and $s_{2}$, given $q_{1}$ and $q_{2}$ (defined exactly by Eq. (5)) is made within the Coulomb cone and thus yields a choice confined to a bounded interval. In the inequalities $(7,8)$, appears a $\operatorname{term} \tan \theta-\mu$ which diverges when $\mu$ gets closer to $\tan \theta$. Figure $2 \mathrm{~b}^{\prime}$ displays a situation where $\mu>\tan \theta$, and from this picture it is clear that the transversal force does not get any upper bound. Therefore, in this case $(\tan \theta<\mu)$, the force scale is not fixed by the problem itself but by the history of the piling preparation. This could be called an hyper-hysteretic problem where the piling history is always relevant to fix the scale of the contact forces. Thus it is clear that beyond this limit, the stochastic approach we aim to built here is bound to fail! In the following we assume to operate below this limit.

\section{Numerical Extraction of the Force Network}

Now we propose a way to built a force network made of vertices of acceptable mechanical solution. An analytical solution based on the previous, set of equations and inequations, would be indeed a difficult task, except in the case where $\mu=0$. This question has been studied by several authors earlier [7,20-22]. Ouagenouni et al. [7] have recently proposed an original approach to study this problem and they have shown that, even for a regular piling, a slight amount of geometrical disorder would lead to a unique non trivial force distribution. Here, we propose to explore the space of solutions with $\mu \neq 0$, using a numerical Monte Carlo method. Note that our kind of regular vertex configuration would lead, in the case $\mu=0$, to no force mixing. Hence, the presence of friction is the only source of disorder here. We extract sets of exact mechanical solutions for the force network using a random choice 
of the parameter $p$ at each vertex within the constraints of macroscopic laws of contact. Note that such a procedure seems arbitrary but is motivated by the true nature of solid-solid contact forces which is a difficult question. Using the Coulomb laws of dry friction, the exact value of the contact force can be shown to depend in a complicated way on the dynamical history of the piling preparation [16]. Since the effect of the history cannot be estimated. we made the assumption that the different initial possible preparations can be rendered by assuming that all $p$ 's could be taken at random without any correlation. Furthermore, it is not clear whether some specific preparation conditions for a granular piling would imply a correlated choice of this parameter field. But we leave this deep question out of the scope of the present paper, we just tackle briefly this issue, on an example, in Sections 5 and 7 . Usually, in molecular dynamics simulations, one assumes that below the coulomb angle, an elastic type of contact prevails [5]. Here, we do not make such a choice. The method we propose bears many resemblance with the contact mechanics algorithm that was initially designed by Moreau et al. [4] and which was used to study the stress distribution in an assembly of polydisperse grains [6].

Other important questions concern the boundary conditions. For the moment, we only consider cyclic lateral boundary conditions supplemented with conditions of a given force at the top coming from a lid (including a zero force, i.e. the free boundary condition). This constraint is implemented by an initial layer with a given vertical charge. For each layer, the vertices are explored starting from a randomly chosen bead following a nearest neighbor progression in a randomly chosen direction. The process is iterated exploring deeper and deeper layers. If a choice of $p$ cannot be made coming from the fact that the inequalities $(6,7,8)$ do not provide any intersection, we use a numerical scheme which converges to a solution rather rapidly when the total number of vertices is not too large (around 5000). The procedure is implemented as follows. If no acceptable solution is found, we move backwards (first in horizontal, then in vertical direction) and try again new sets of $p$ 's chosen at random. To improve the search speed, the number of steps we move backwards for each failure case increases with the number of failures. In addition, this number of steps is initialized each time the algorithm reaches a depth beyond the maximum depth previously reached. This scheme eliminates rapidly unwanted solutions. Thus, a large number of networks can be extracted and this provides a statistical ensemble of realizations. Note that it is not clear mathematically whether the Monte Carlo method described above is the most efficient to explore thoroughly the solution space. This question is linked to the topological structure of the solution space, another difficult question indeed. As it is usually done in the standard Monte Carlo method, one could introduce a cost function to extract from all possible solutions the one with the lowest "temperature". But since the effect of a real experimental preparation on the distribution of the $p$ 's is unknown, the definition of a cost function would be hazardous. We rather keep all mechanically possible events without introducing any weight on any of them other than the weight inherent to the numerical algorithm used. The aim of the next chapters is to explore the statistical properties of the network realization ensemble hence constructed and to define a bridge with stochastic models.

\section{Structural and Stochastic Properties of the Force Networks}

In this Section, we investigate some structural properties of the force networks, we have generated. We are mostly interested in the vertical force transport properties i.e. the vertical charge $C$. In Figure 3, we show three examples of networks of size $50 \times 100$, extracted with three different coefficients of friction: $\mu=0.1, \mu=0.4$ and $\mu=0.6$. We monitor the relative fluctuations with respect to the average charge $\langle C(z)\rangle$ (which grows linearly with the depth $z$ ). The grey scale is set to display the largest rescaled forces $f=C /\langle C(z)\rangle$ as the darkest 
a)

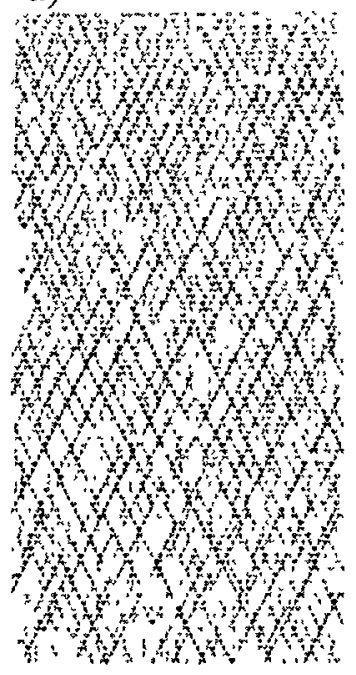

b)

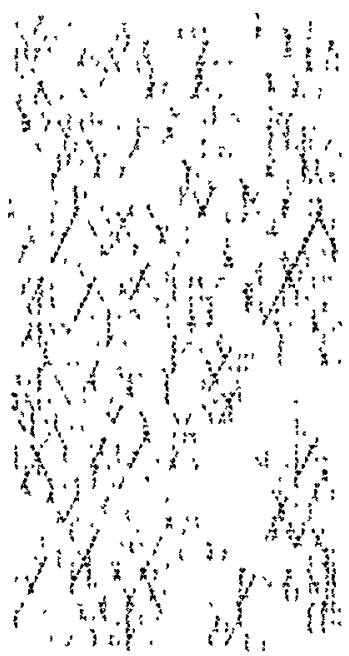

c)

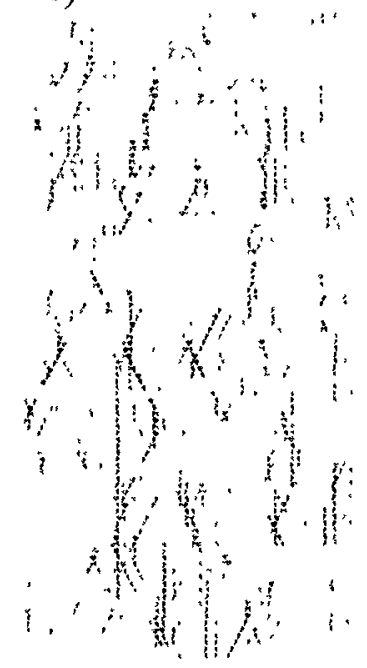

Fig. 3. - Vertical charges distributions for a $50 \times 100$ cyclic piling. We displayed a single realization for three static friction coefficients $\mu$. The grey scale represents the rescaled charge $f$ (as defined in the text). The darkest tones are the larger forces: a) $\mu=0.2$, b) $\mu=0.4$, c) $\mu=0.6$.

tones. We observe clearly the presence of large force paths along lines at $\theta=60^{\circ}$ and $\theta=120^{\circ}$ just like in the experiments on regular packing by Travers et al. [2]. The use of a large friction coefficient yields a structure for which the direction of propagation of the large forces paths is much more disordered. In Figure 4, we report the distribution of the vertical charge for various friction coefficients. We display on a Log-normal scale, the force distribution $P(f)$, rescaled such that $\int P(f) \mathrm{d} f=\int f P(f) \mathrm{d} f=1$. We choose such a rescaling of the probability distribution function in order to be able to compare directly our results with the mean field distribution proposed by Liu et al. [12]. This normalization yields an universal shape for $P(f)$ independent of the depth at sufficiently large depths.

Furthermore, we establish a direct comparison with stochastic models presented in reference [12]. We define the quantities $\widetilde{q}_{1}=q_{1} / C$ and $\widetilde{q}_{2}=q_{2} / C$ which are the fractions of total vertical charge redistributed on each contact downwards. Liu et al. [12] have suggested that the redistribution fractions $\widetilde{q}_{1}$ and $\widetilde{q}_{2}$ could be taken from a flat distribution $\Pi(\widetilde{q})=1$ in the interval $[0,1]$. In the context of their model, a mean-field analytical solution (for a number of contact per vertex $N=2$ ), yields the following force distribution:

$$
P^{\mathrm{MF}}(f)=4 f \exp (-2 f)
$$

However, we observe in Figure 4 that the distribution $P(f)$ is modified by the value of $\mu$ and, in the limits of the numerical capacities, we could never obtain a distribution similar to the meanfield distribution $P^{\mathrm{MF}}(f)$. Also, we monitored the first moment $\sigma_{\mathrm{f}}$ of the force distribution and we observed that the saturation of this first moment takes a longer depth for lower friction coefficients. But an important point to consider is that the value of the average fluctuation is always of the order of magnitude of the average force. This result is consistent with many measurements, either experimental [12] or numerical [6,7] obtained for different friction and geometry conditions. Nevertheless, contrarily to those previous claims we never really got 


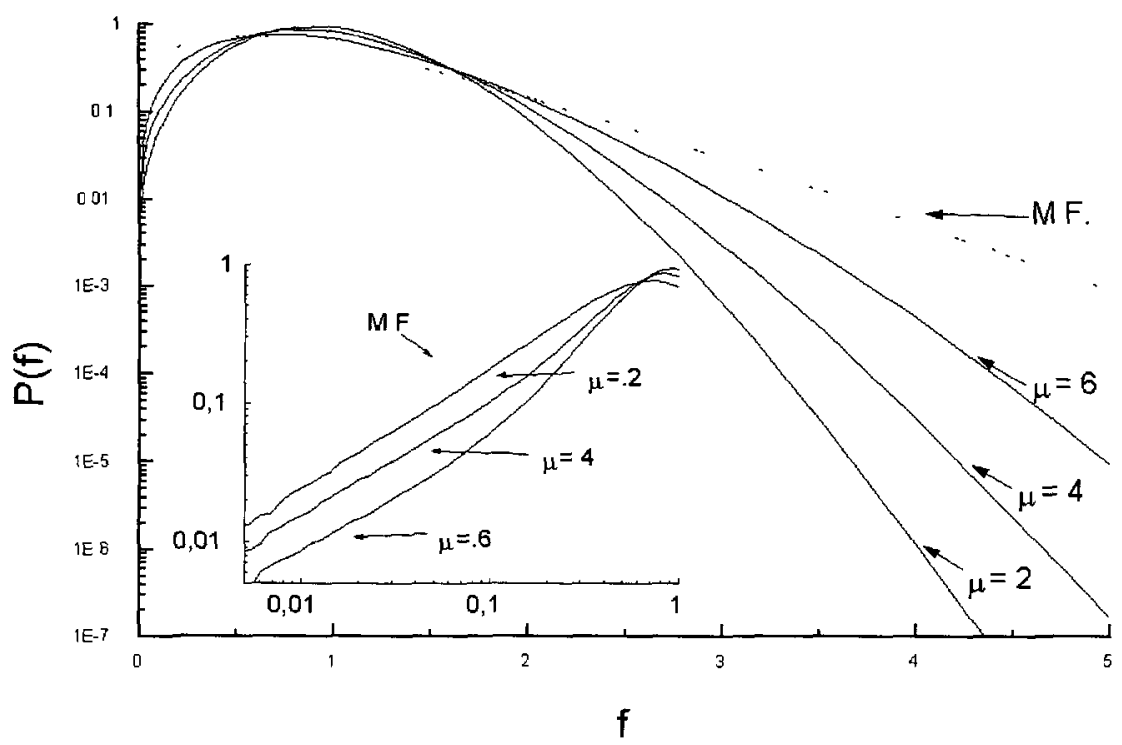

Fig. 4. - Rescaled charge distribution $P(f)$ for different values of the friction coefficient $\mu$. The statistics were made over 10,000 pilings for each friction coefficient. The geometry used is $15 \times 1000$ $(15 \times 2000$ for $\mu=0.2)$ and we only considered the bottom half of the piling to extract the distribution $P(f)$. The inset is the small forces distribution in a log-log scale. The dashed line is the Mean Field solution of equation (9).

an exponential decrease for large forces as the model of Liu et al. would fundamentally predict. An extension of the theory of Liu et al. [12] has been proposed [23] dealing with a probability distribution of the relative charges, $\Pi(\widetilde{q})$ such that $\Pi(\widetilde{q}) \neq 0$ when $1-\widetilde{q}_{\max }<\widetilde{q}<\widetilde{q}_{\max }$ and $\Pi(\widetilde{q})=0$ otherwise. Note that $\widetilde{q}_{\max }=1$ corresponds to the original theory of Liu et al. [12]. A central result of this scalar transport theory is that the force distribution should decay as:

$$
P(f) \sim \exp \left(-f^{\alpha}\right) \text { with } \alpha=\frac{\log N}{\log N-\log \left(1 / \widetilde{q}_{\max }\right)} .
$$

The distributions tails of Figure 4 can be fitted with an apparent $\alpha$. For example, we measured $\alpha(\mu=0.2)=1.77 \pm 0.01, \alpha(\mu=0.4)=1.50 \pm 0.01$. A direct look at the $\Pi(\widetilde{q})$ distribution (see Fig. 5) does not corroborate the existence of a $\widetilde{q}_{\max }$ cut-off. This result is an indication of another class of universality for the charge propagation statistics in the presence of solid friction. It is likely to be a consequence of the underlying vectorial character of the force equilibrium problem; we reconsider this issue on another perspective in Section 6.

Now we intend to provide a qualitative physical picture of the network organization by monitoring the problem in terms of charge collection of two incoming charges. A qualitative description of the situation encountered at each vertex where two charges cross, is sketched in Figure 6. For a given bias (charge distribution unbalanced at the top) either, the larger force collects the small one (Fig. 6a) or redistributes a fraction evenly of its value (Fig. 6b) or even anti-collect (Fig. 6c). Such a situation is captured by the parameter $\beta=\frac{q_{2}-q_{1}}{Q_{2}-Q_{1}}$ such that $\widetilde{q}_{1}=\frac{1}{2}+\frac{\beta}{2} \frac{\left(Q_{2}-Q_{1}\right)}{C}$ Hence $\beta>1$ represents a charge collection, $0<\beta<1$ a redistribution and $\beta<0$ an anti-collection. Recently more stochastic models of force propagation have 


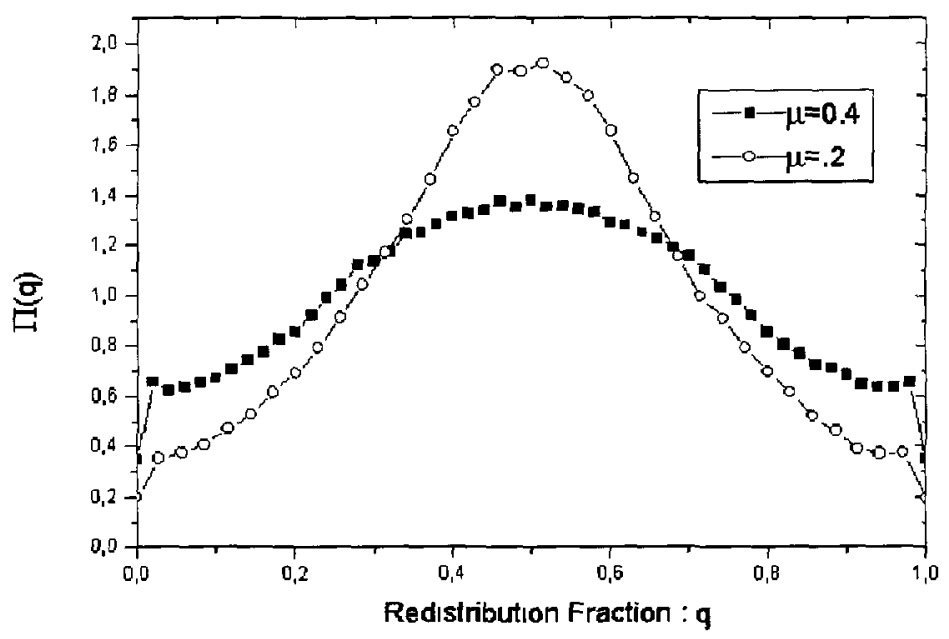

Fig. 5. - Probability of the vertical charge redistribution fraction $\Pi(\widetilde{q})$, calculated on a $25 \times 100$ lattice at a depth $z=100$ for two friction coefficients $\mu=0.2$ and $\mu=0.4$.

a)

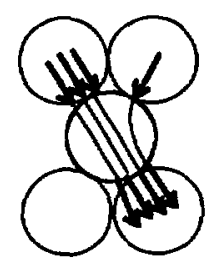

$\beta>1$ b)

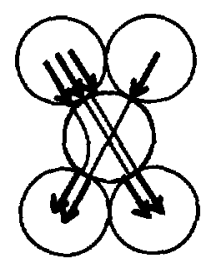

$0<\beta<1$ c)

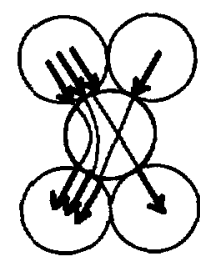

$B<0$

Fig. 6. - Interpretation of the collection parameter $\beta$ in terms of crossing vertical charges; $\beta>1$ is a "collection", $0<\beta<1$ is a "redistribution" and $\beta<0$ is an "anti-collection".

been proposed $[14,15]$, which includes effectively a parameter of the type of $\beta$. These models basically assume the capture of small charges by large charges, whenever the charges received on the top on a bead is strongly unbalanced. This case would correspond to a situation where $\beta>1$. In Figure 7 , we display the average value of the parameter $\langle\beta\rangle$ as a function of the rescaled charge $f$ for three values of the friction coefficient $\mu=0.2, \mu=0.4$ and $\mu=0.6$. Note that, in order to avoid a undesired divergence of $\beta$, we calculated the averaged $\beta$ for $\left|Q_{1}-Q_{2}\right| /\left(Q_{1}+Q_{2}\right)$ larger than 0.1 only. Clearly, the average tendency is a charge redistribution but an interesting self-organization property is evidenced: it seems that smaller charges tend to cross each other with a weak tendency to redistribute but large charges show a net tendency to redistribution. A transition to anti-collection properties is even possible for larger friction (it would corresponds to a negative $\langle\beta\rangle$ ). Actually, we also noticed that the distribution of $\beta$ 's shows a large standard deviation (of the order of 1 ). It is likely that families of solutions can be found within the global space of solutions that would allowed collection of the larger forces, due to specific local conditions on the choice of parameter $p$. This is the type of question we address in the next chapter on one example. 


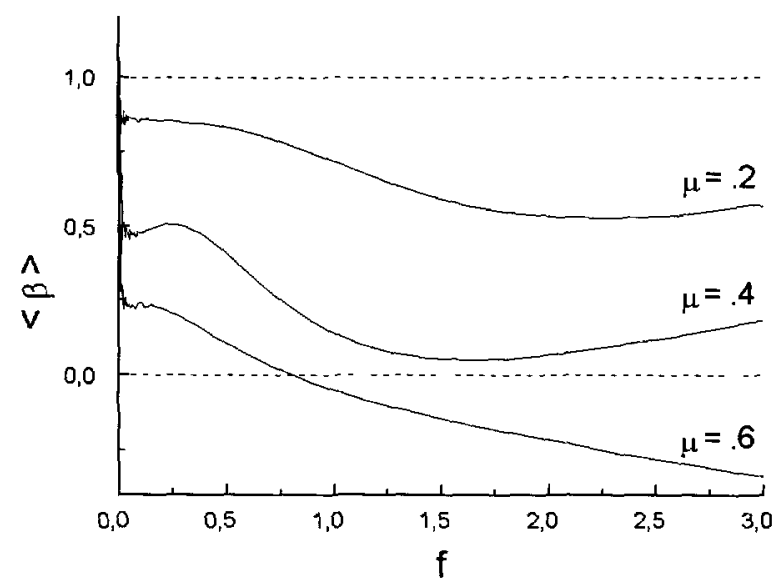

Fig. 7. - Average collection parameter $\langle\beta\rangle$ as a function of the rescaled vertical charge $f$ for three friction coefficients, $\mu=0.2, \mu=0.4$ and $\mu=0.6$. We used the same runs as in Figure 4 keeping only the beads with a significative charge difference on the top i.e. $\left|Q_{1}-Q_{2}\right| /\left(Q_{1}+Q_{2}\right)>0.1$.

\section{Effect of a Local Bias on the Parameter Choice}

Now we explore the possibility to extract subsets of acceptable mechanical solutions which will include auto-organization of the force network with stochastic properties drastically different from the previous situation. The approach we present here may seem arbitrary since we cannot say anything on dynamical processes that could lead the piling to the described situation. Nevertheless, this example is developed to illustrate the importance of having a pertinent description of the granular contacts force status as well as the dynamics leading to equilibrium since, as we will demonstrate, the macroscopic picture can be strongly influenced by a local "polarization" of the friction forces.

We notice that the collection parameter previously defined may be written:

$$
\beta=-1+2 \sqrt{3} \frac{S_{1}+S_{2}}{Q_{1}-Q_{2}}
$$

its value is solely determined by the choice of the stochastic parameters from the layer just above. The idea is to find some rule that could maximize $\beta$, in the average, in order to favor a force collection (if possible). We make the following remark: when the friction is not too large ( i.e. $\mu<1 / \tan \theta$ ), we have $S_{1}>0$ and $S_{2}<0$ (see in Fig. 1 for sign convention). When two charges with a significative difference in amplitude cross at a vertex, one can neglect the smallest one. Making this assumption, $\beta$ is maximized by maximizing the quotient $\left|S_{\imath}\right| / Q_{2}$ where the subscript $i$ stands for the largest charge. A way to achieve this goal ( $\beta$ maximum) would be to maximize this quotient for all the beads. This can be done by orientating the friction forces in a way sketched in Figure $8 \mathrm{a}$. In this picture, we sketched and orientation of the friction forces in a preferential fraction of the coulomb cone (see the shaded area). On this example (if we suppose that $Q_{2}$ is the largest charge), the quotient $S_{2} / Q_{2}$ is maximized for a projection of the tangential contact force in the part of the coulomb cone just below the shaded area. The same reasoning holds when $Q_{1}$ is supposed to be the largest charge and therefore the tangential contact force directions should be as sketched in Figure 8a. Similarly, a situation like in Figure $8 \mathrm{~b}$ represents the opposite situation where $\beta$ would be minimum. Following 


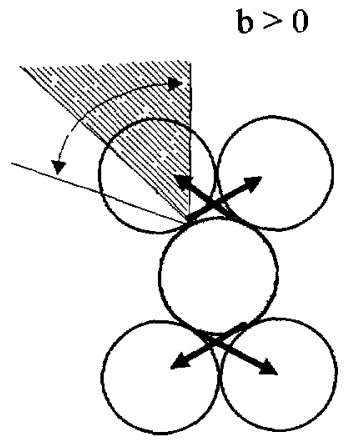

(a)

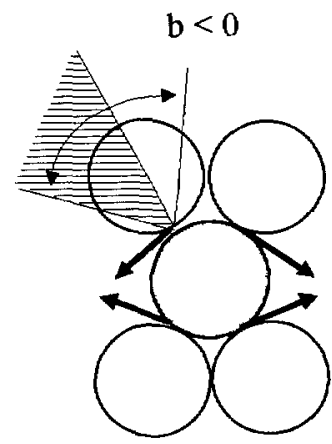

(b)

Fig. 8. - Sketch of the direction of friction forces transmitted on the central bead. The fraction of the Coulomb cone preferentially used for the upper bead 2 is shaded a) case where $b$, bias parameter, is positive (this geometry favor collection of the forces) and b) case where $b$ is negative.

this remark, we design an algorithm where the choice of $p$ is such that only a fraction of the Coulomb cone is selected in order to produce situations like in Figure 8. Hence, we introduce a bias parameter $b$ such that $-2<b<2$ which characterizes the available fraction of the Coulomb cone for the contact forces. Parameter $b$ is defined according to the rule:

$$
\begin{aligned}
& -\mu\left(1-\frac{b+|b|}{2}\right) n_{1}<t_{1}<\mu\left(1+\frac{b-|b|}{2}\right) n_{1} \\
& -\mu\left(1+\frac{b-|b|}{2}\right) n_{2}<t_{2}<\mu\left(1-\frac{b+|b|}{2}\right) n_{2} .
\end{aligned}
$$

The parameter $b$ constraints a situation like Figure $8 \mathrm{a}$ when positive and like Figure $8 \mathrm{~b}$ when negative (see in Fig. $1 b$ for surface forces, $t_{\imath}$ and $n_{\imath}$, definitions). Note that the case $b=0$ corresponds to the previously studied case of a projection in the full Coulomb cone. In Figure 9 we display the result of the average collection parameter calculated for three values of the bias parameter: $b=0$ and $b= \pm 1.275$. Now it is clear that the case $b>0$ corresponds in the average to a positive collection parameter $(\beta>1$ for all values of the charges). Therefore in this case, large charges will collect up small ones. In Section 7 , we will study this effect influence on the force distribution at the bottom of a sand-pile.

\section{Response to a Localized Force}

In this study, we set the gravity to zero and we prepare the lid with a given charge of value $C_{0}$ on each bead, except one bead which bears the value $C_{\mathrm{o}}+\delta C_{\mathrm{o}}$. In this chapter simulations are performed with no bias condition of the friction forces. We observed that generically the maximum amplitude of the response propagates as a "light ray" with a direction of $\theta=60^{\circ}$ and $120^{\circ}$ with respect to the horizontal (See Fig. 10a). This result is qualitatively consistent with the results coming from the closure conjecture of the mechanical equations proposed by Bouchaud et al. [8]. Nevertheless, we observe a loss per unit length and a dispersion of 


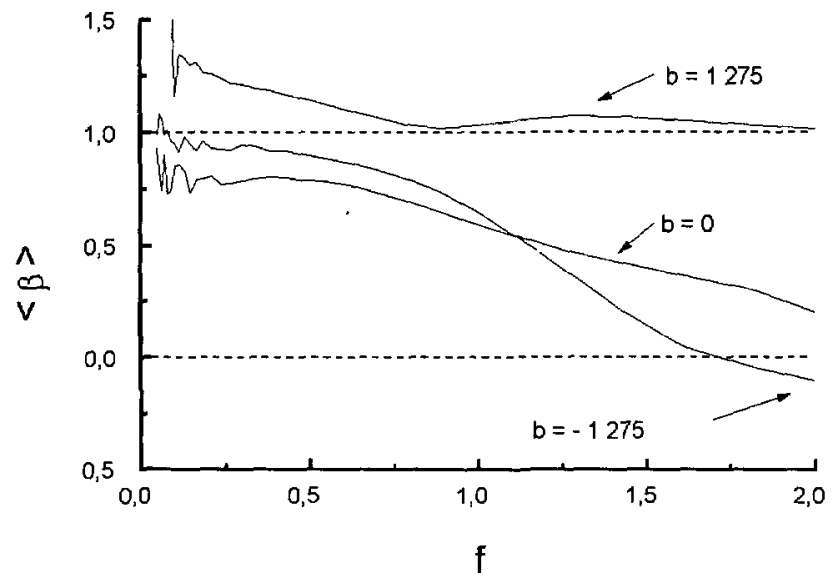

Fig. 9. - Average collection parameter $\langle\beta\rangle$ as a function of the rescaled vertical charge $f$ for three different values of the bias parameter $b: b=-1.275, b=0$ and $b=1.275$. The statistics were made on the bottom half of 10,000 pilings, for each $b$, with $\mu=0.2$. Note that we used a rather small geometry (a $15 \times 50$ grid) because the case $b$ positive is costly. This is why the case $b=0$ does not match the asymptotic value obtained in Figure 7.

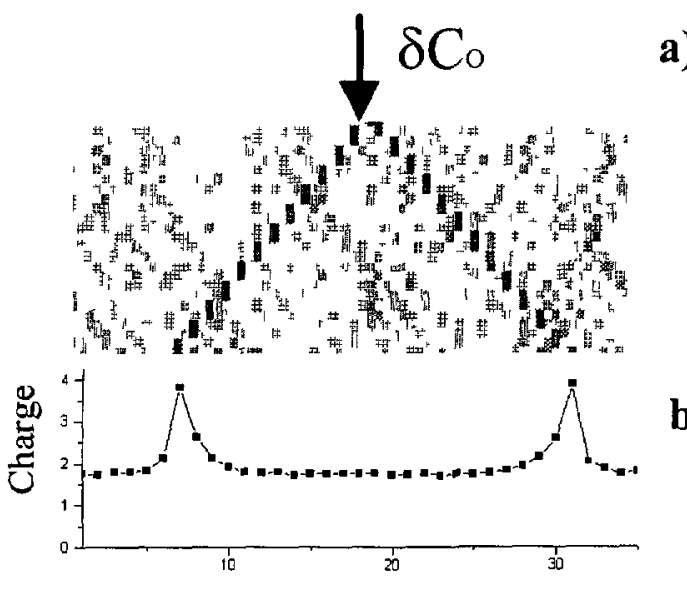

a)

Horizontal Distance : $\mathrm{x}$

Fig. 10. - Response to a localized force. a) Vertical charge distribution for a single realization. b) Average distribution of the vertical charge at the depth $z=25$.

the force on the neighbors which yields a shape as represented in Figure 10b. Note that we did not monitor the response up to very large depths (only $z=25$ ). But already, we have indications on general features of the transport properties. From these data, we extract $\kappa$, a loss coefficient per unit length. In the top layer, the original excess force is lost simply by possibility of compensation of the two ray going away from each other, so, it depends solely on the way we press on the material. The features of the loss are very different from a simple diffusion. The first feature is that the shape of mass excess is not symmetrical around the light 
(a)

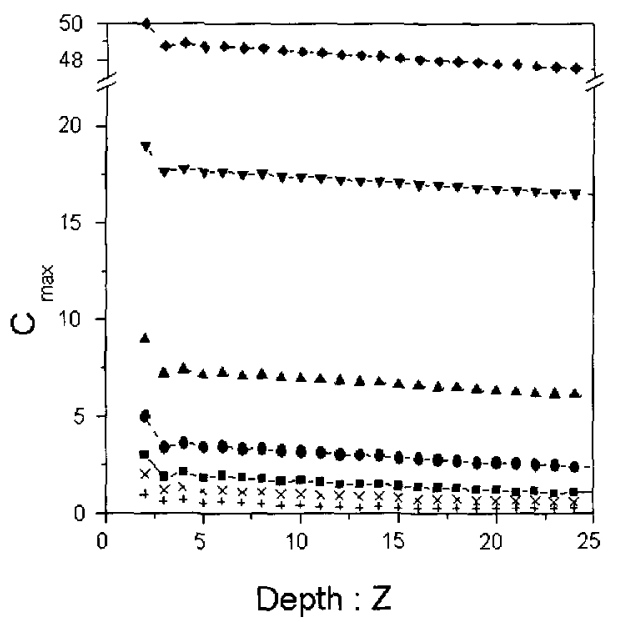

(b)

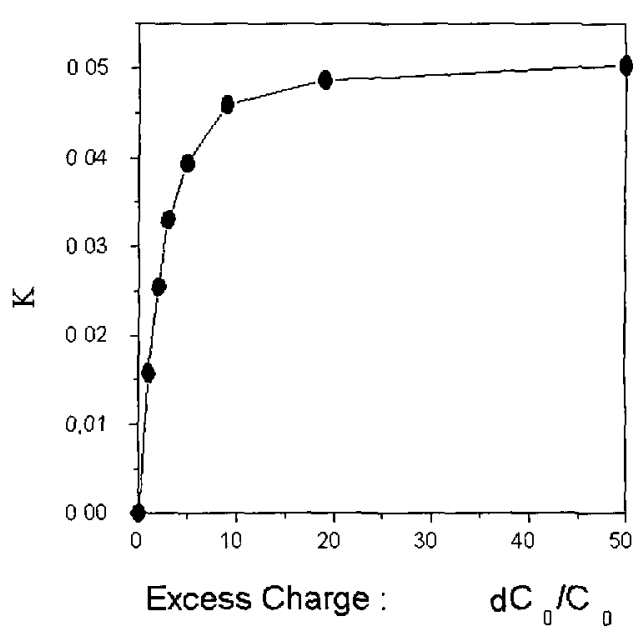

Fig. 11. - a) Evolution of the maximum of the average vertical charge $C_{\max }$ with the depth for initial values of $\delta C_{\circ} / C_{o}=50(\bullet), 20(\mathbf{\nabla}), 10(\boldsymbol{\bullet}), 7(\bullet), 5(\boldsymbol{\square}), 3(\times), 2(+)$. The loss coefficient $\kappa$ obtained from the linear fit is reported on b) as a function of $\delta C_{\mathrm{o}} / C_{\mathrm{o}}$.

rays (Fig. 10b). This result could be compared to the model of Bouchaud et al. [8]. It can be understood considering that only the inside of the cone is sensitive to the mass excess $\delta C_{0}$. Second, a loss coefficient can be extracted using the straight line fit visible in Figure 11a. In Figure $11 \mathrm{~b}$, we report the loss $\kappa=\partial\left(C_{\max }\right) / \partial z$, for a fixed friction $\mu=0.4$ as a function of $\delta C_{\mathrm{o}}$. We observe a strong non-linearity in the behavior of this coefficient. For large $\delta C_{0} / C_{o}$, the loss rate is almost a constant. For the behavior displayed in Figure 11b, we propose an heuristic explanation. In the limit of weak pressure increase, we have a force excess comparable to the average fluctuating background and the mixing laws are in first approximation, compatible with an average collection parameter $\bar{\beta}$ which gives a linear behavior at low $\delta C: \kappa \approx(1-\bar{\beta}) \delta C_{\mathrm{o}}$. But for large values of $\delta C_{\mathrm{o}} / C_{\mathrm{o}}$, the saturation of the loss rate is due to the fact that large forces cannot use the whole coulomb cone to redistribute in the network. Consequently, the loss is always of the order of the average fluctuating background. This is a direct consequence of the local rotation force balance. Note that this kind of behavior involving torque balance at the level of a single grain is absent from standard coarse-grained mechanical equation description. It is also clear that in a real situation, the higher is the force excess, the most likely is the probability for the system to vield and create a dynamical deformation. This situation is of course not taken into account in the modelization.

\section{The Sand-Pile}

Now we look at the force distribution below a sand pile. The pile we prepare is a regular cannon ball piling with a $\phi=30^{\circ}$ angle slope. Theoretical results $[20,21]$ show for $\theta=60^{\circ}$, on a regular frictionless piling, that the vertical force distribution should be linear on the edges and show a plateau in the inside. An experimental work has shown the possibility in 3D of a dip at the pressure plateau limit due to self organization of the force network [24]. Some simulations have shown that a dip may exist on a frictionless piling but might be due to 


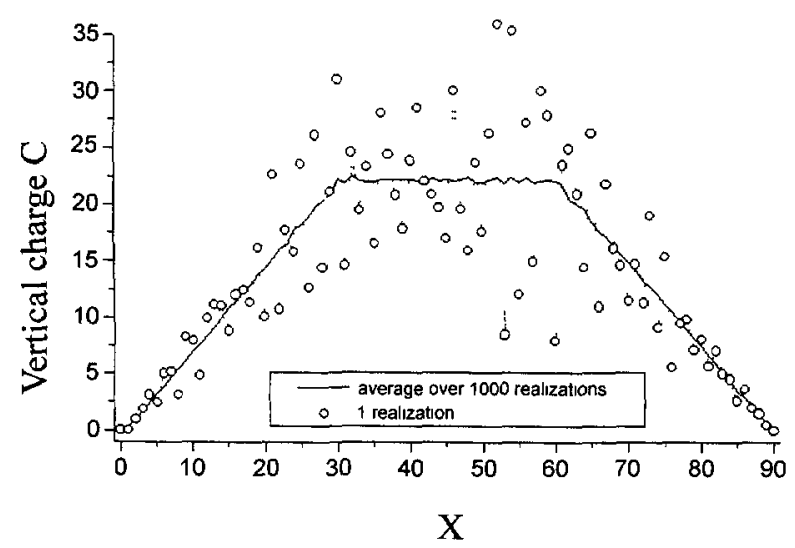

Fig. 12. - Distribution of the vertical charge at the bottom of a sand pile of $30^{\circ}$ angle slope. $\mu=0.2$.

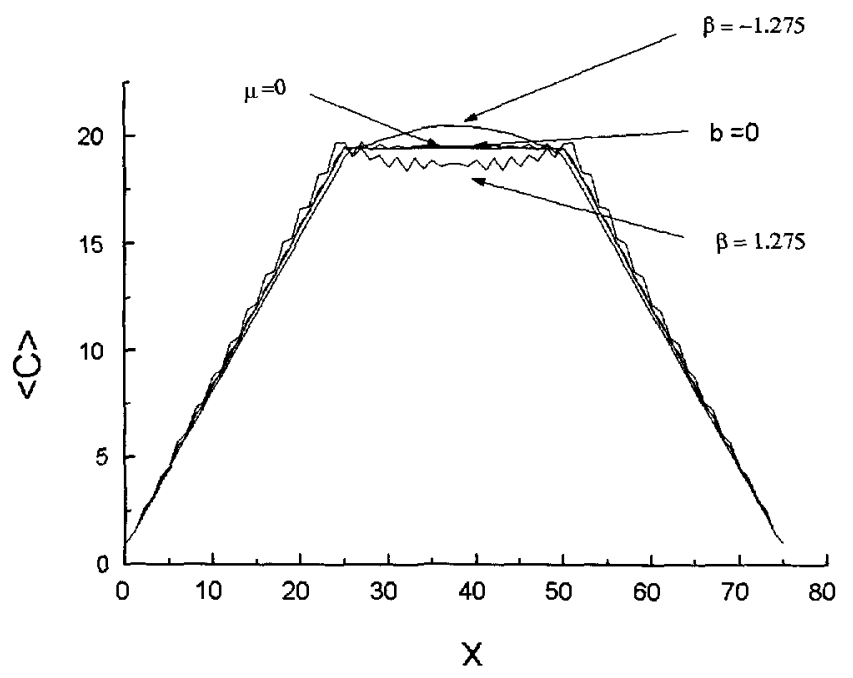

Fig. 13. - Distribution of the vertical charge at the bottom of a sand pile for three different values of the bias parameter $b$ with a friction coefficient $\mu=0.2$. The average is taken over 100,000 pilings. A comparison is made with a piling for which $\mu=0$. Fluctuations, not represented here, are of the order of 2 dimensionless units of charge in the three cases.

a geometrical organization of the contact network [22]. Using the method previously described, we performed series of simulations and found no evidence for a dip of in the middle of the heap. We find a high level of fluctuations as evidenced on the distribution of pressure on one example of the piling (see Fig. 12) but, on the average, the pressure distribution corresponds to a frictionless piling.

Nevertheless, we find that it is possible to depart from a straight plateau in the context of our model piling if the heap is prepared with a specific condition on the friction forces. The situation we study here is the friction biased condition presented earlier in chapter 5 . In Figure 13, we display the vertical charge distribution at the bottom of a sand pile prepared 
with a $\phi=30^{\circ}$ angle slope. From the picture we observe that, when the bias parameter $b$ goes from negative to positive values, the charge distribution in the central part of the pile goes from a force excess (a bump) to a force deficit (a dip). Now, we explore if this change of behavior, associated to local prescriptions on the friction forces, can be also understood in terms of macroscopic constitutive relations. In a $2 \mathrm{D}$ situation like ours, the mechanical equations involve the four components of the stress tensor combined in a way that any macroscopic volume element is stable vertically and horizontally i.e.

$$
\begin{aligned}
& \frac{\partial \sigma_{z z}}{\partial z}+\frac{\partial \sigma_{x z}}{\partial x}=\rho_{\mathrm{o}} g \\
& \frac{\partial \sigma_{z x}}{\partial z}+\frac{\partial \sigma_{x x}}{\partial x}=0
\end{aligned}
$$

where $\rho_{0}$ is the granular density and $\sigma_{\imath \jmath}$ are the usual stress tensor components. These two equations associated with the condition that the elementary volume does not rotate r.e. $\left(\left|\sigma_{x z}\right|=\left|\sigma_{z x}\right|\right)$ will form a set of three equations for four unknowns which are the mesoscopic equivalent of equation (4) at the granular level. Clearly, to get a solution of the problem, one needs a constitutive relation linking together components of the stress tensor. A proposition was recently made by Bouchaud et al. [8], based on simple symmetry and scaling considerations, they proposed an extension of Janssen's [25] constitutive relation in the form:

$$
\sigma_{x x}=K \sigma_{z z}+\frac{\bar{\lambda}}{\sigma_{z z}} \sigma_{x z}^{2}+\frac{\overline{\lambda^{\prime}}}{\sigma_{z z}} \sigma_{z z}^{2}+. .
$$

where coefficients $\bar{\lambda}$ and $\overline{\lambda^{\prime}}$ are dimensionless quantities. From the previous set of data for the vertical and horizontal forces at a vertex, we measure the values of the stress tensor, using the relations (the average is made over thousands of realizations):

$$
\begin{aligned}
\sigma_{x x} & =\frac{1}{4}\left(N_{1}+N_{2}\right)-\frac{\sqrt{3}}{4}\left(T_{1}-T_{2}\right) \\
\sigma_{z z} & =\frac{3}{4}\left(N_{1}+N_{2}\right)+\frac{\sqrt{3}}{4}\left(T_{1}-T_{2}\right) \\
\sigma_{x z} & =\sigma_{z x}=\frac{\sqrt{3}}{4}\left(N_{1}-N_{2}\right) .
\end{aligned}
$$

To test a constitutive relation like equation (14), we display $\sigma_{x x} / \sigma_{z z}$ as a function of $\left(\sigma_{x z} / \sigma_{z z}\right)^{2}$ in Figure 14: we represented the results for three values of $b$ (the values of Sect. 5 i.e. $b=0$ and $b= \pm 1.275$ ). In the case $b=0$, we have a constant value $K \simeq 0.34$, corresponding to Janssen's type constitutive relation. This numerical value is very closed to $K=1 / 3$ which would be the theoretical expectation [8] for a force propagation at a direction $\alpha=30^{\circ}$ with respect to the vertical axes as evidenced in Section $6: K_{\text {theor }}=(\tan \alpha)^{2}=1 / 3$. For $b \neq 0$, the straight slopes on the graph, witness a constitutive relation consistent with equation (14). In first approximation it seems that $\bar{\lambda} \sim-b$ and $\overline{\lambda^{\prime}} \sim-b$. Note also that this result is also in full agreement with the analytical result of Bouchaud et al. [8] on the sand-pile stress distribution where the case $\bar{\lambda}>0$ would correspond to a bump and $\bar{\lambda}<0$ would correspond to a dip.

\section{Conclusion}

In this paper, we present a numerical approach suited to study the forces distribution in a static granular piling. We show explicitly that the mechanical equation describing local static 


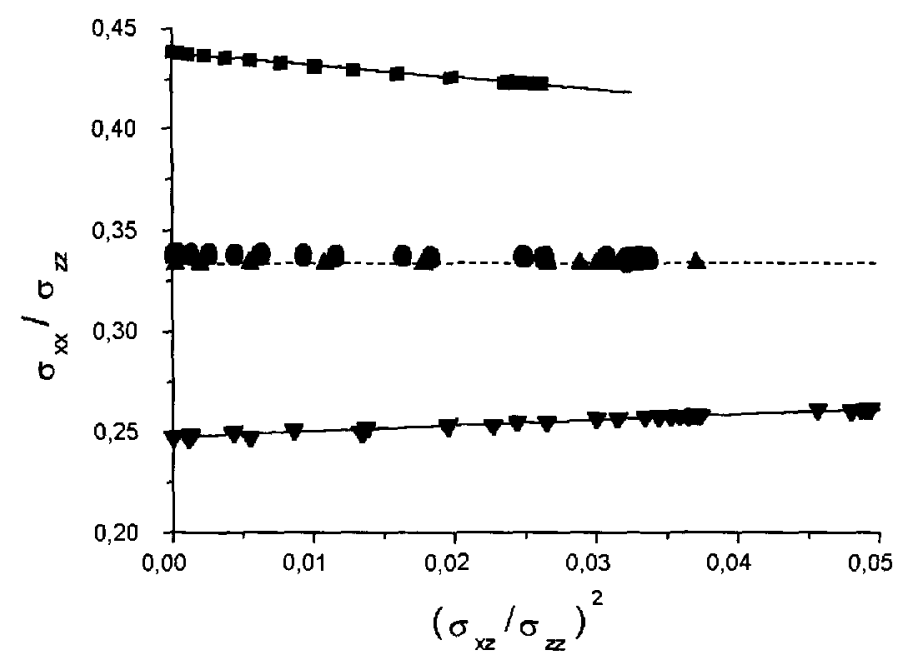

Fig. 14. - Plot of $\sigma_{x x} / \sigma_{z z}$ as a function of $\left(\sigma_{x z} / \sigma_{z z}\right)^{2}$ for $b=-1.275(\mathbf{\square}), b=0(\bullet), b=1.275(\boldsymbol{\nabla})$ and $\mu=0(\boldsymbol{\Delta})$. We used the same runs as in Figure 13 .

equilibrium can be related to a stochastic variable representing the free choice for the friction forces within the Coulomb cone. We design a Monte Carlo algorithm in order to solve this problem in the case where, from site to site, the choice of acceptable mechanical solution is made randomly within all the available possibilties. We observe that for such a simple piling geometry, a non-trivial organization is present. The geometrical properties of this self-organized network induce a spontaneous creation of privileged paths transporting the larger forces. A large friction coefficient increases the disordered character of the force paths. Measurements on the distribution of charges at the bottom of the layers indicate that the fluctuation of the charge is of the same magnitude than the average itself. A statistical analysis shows that the transport properties of the vertical charge cannot be rendered using a simple scalar stochastic model. Moreover, we show that in the average there is no collection of smaller forces by larger forces when they cross at a vertex but rather, a mere redistribution, small forces gaining and large ones loosing. However we show that biasing the projection of the friction forces within a fraction of the Coulomb cone can have a drastic macroscopic outcome: larger forces can gain in amplitude when they meet smaller forces at a vertex for a specific orientation of the friction forces. We call this phenomenon charge collection. Furthermore, we study the response of a single pressure step and we show that it propagates along straight lines with a rate of loss depending, in a non linear way, on the pressure increase.

Thus, on this simple static mechanical model, we show that the force propagation is still a complicated issue and, maybe, a stochastic vision of it should imply full consideration of the underlying vectoral nature of the equilibrium mechanics. What is not clear yet, is whether additional geometrical disorder has the possibility to change drastically this picture and maybe simplify the transport properties as Liu et al. [12] have suggested.

In addition, we observe that the model we use does not lead, in the average, to a pressure hole below a sand pile. Nevertheless, we may observe something different than a plateau in the central part of the pile if we prepare the system using a bias on the local orientation of the friction forces. Remarkably we verified that, in this locally biased situation, the stress 
closure equation is modified and is consistent with the macroscopic conjecture of Bouchaud et al. [8] and we obtain either a force excess or a force dip according to the sign of our local bias. Also, when the friction forces are locally biased, our fully mechanical model can be linked to cellular automaton results describing force propagation in a granular assembly $[14,15]$. Note also that, in our simple model, the physical interpretation for the charge collection phenomenon is different from what Hemmingson et al. [14] or Claudin et al. [15] have originally suggested (loss of contact between grains). Finally, this work indicates the necessity of a finer description for the solid on solid interactions between grains including the study of the relevant dynamical aspects of the contact forces near static equilibrium in connection with contact network dynamics.

\section{Acknowledgments}

We thank Prof. P.G. de Gennes for an insightful seminar series at the Collège de France and J. Duran, J. Rajchenbach and J.P. Bouchaud for many discussions.

\section{References}

[1] Dantu P., in Proc. 4th Int. Conf. Soil Mechanics and Foundation Engineering (Butterworth Scientific Publications, London, 1957).

[2] Travers T., Bideau D., Gervois A. and Messager J.C., J. Phys. A 19 (1986) L1033.

[3] Cundall P.A. and Strack O.D.L., Geotechnique 29 (1979) 1, 47.

[4] Moreau J.J., Eur. J. Mech. A Solids 13 (1994) 93.

[5] Zhuang X., Didwania A.K. and Goddard J.D., J. Comp. Phys. 121 (1995) 331.

[6] Radjai F., Thèse de l'Université Paris XI (1995); Radjai F., Jean M., Moreau J.J. and Roux S., Phys. Rev. Lett. 77 (1996) 274.

[7] Ouaguenouni S. and Roux J.N., Europhys. Lett. 32 (1995) 449; Ouaguenouni S. and Roux J.N., Europhys. Lett. 39 (1997) 117.

[8] Bouchaud J.P., Cates M.E. and Claudin P., J. Phys. I France 5 (1995) 6389.

[9] Edwards S.F. and Mounfield C.C., Physica A 226 (1996) 1.

[10] Wittmer J., Claudin P.. Cates M. and Bouchaud J.P., Nature 382 (1996) 336.

[11] Nedderman R.M., Statics and Kinematics of Granular Materials (Cambridge University Press, 1992).

[12] Liu C.H., Nagel S.R., Schecter D.A., Coppersmith S.N., Majumdar S., Narayan O. and Witten T.A., Science 269 (1995) 513.

[13] Coppersmith S.N., Liu C.H., Majumdar S., Narayan O. and Witten T.A., Phys. Rev. E 53 (1996) 4673.

[14] Hemmingsson J., Herrmann H.J. and Roux S., J. Phys. I France 7 (1997) 291.

[15] Claudin P. and Bouchaud J.P., Phys. Rev. Lett. 78 (1997) 231.

[16] Mindlin R.D. and Deresiewicz H., J. Appl. Mech. (Trans. A.S.M.E.) 20 (1953) 327.

[17] Caroli C. and Nozière P., in "Physics of Sliding Friction", B.N.J. Person and E. Tossato, Eds., NATO ASI series, Appleed Sciences, Vol. 311 (Kluwer Acad. Publ., Dordrecht, 1996). 
[18] A preliminary version of the model has already been presented: Clément E., Eloy C., Rajchenbach J. and Duran J., to be published in "Lectures on Stochastic Dynamics", Lecture Notes in Physics Series, T. Poeschel and L. Schimansky-Geier, Eds. (Springer Verlag, Berlin, 1997).

[19] de Gennes P.G., Lecture Notes, Varenna Summer school on Complex systems (July 1996) to be published by Societa Italiana di Fisica.

[20] Liffman K., Chan C.Y. and Hughes B.D., Powder Technol. 72(1992) 255 .

[21] Hong D.C., Phys. Rev. E 47 (1993) 760.

[22] Luding S., Phys. Rev. E 55 (1997) 4720.

[23] Bouchaud J.P., Claudin P., Cates M.E. and Wittmer J., in preparation.

[24] Smid J. and Novosad J., I. Chem. E. Sympos. Ser. 63, D3/V/1 (1981).

[25] Janssen H.A., Zeits Vereins Deutsch Ing. 39 (1895) 1045. 